\title{
Membrane-Associated Guanylate Kinase Proteins MPP4 and MPP5 Associate with Veli3 at Distinct Intercellular Junctions of the Neurosensory Retina
}

\author{
HEIDI STÖHR, ${ }^{1 *}$ LAURIE L. MOLDAY, ${ }^{2}$ ROBERT S. MOLDAY, ${ }^{2}$ \\ BERNHARD H.F. WEBER, ${ }^{1}$ BERND BIEDERMANN, ${ }^{3}$ ANDREAS REICHENBACH, ${ }^{3}$ \\ AND FRANZISKA KRÄMER ${ }^{1}$ \\ ${ }^{1}$ Institut für Humangenetik, Biozentrum, Am Hubland, Universität Würzburg, \\ 97074 Würzburg, Germany \\ ${ }^{2}$ Department of Biochemistry and Molecular Biology, University of British Columbia, \\ Vancouver, British Columbia V5T 1Z3, Canada \\ ${ }^{3}$ Paul-Flechsig-Institut für Hirnforschung, Universität Leipzig, 04109 Leipzig, Germany
}

\begin{abstract}
MPP4 and MPP5 are closely related members of the p55-subfamily of membrane-associated guanylate kinases (MAGUKs) known to mediate the assembly of protein complexes at the plasma membrane of cell-cell junctions. Both MPP4 and MPP5 have been implicated in retinal function; however, their specific roles in the cellular mechanisms underlying vision are largely unknown. Here, we generated specific poly- and monoclonal antibodies against the two proteins and show that MPP4 and MPP5 are localized at distinct sites of cell-cell contact in the mouse retina. While MPP4 is a component of the synaptic terminals of photoreceptors, MPP5 exclusively localizes to apical membrane domains of the outer limiting membrane (OLM) junctions. The vertebrate homologs of Caenorhabditis elegans lin-7, Veli1, -2 , and -3 , have previously been identified as putative binding partners of MPP5. In this study, we show that MPP4 directly interacts with the Veli proteins via L27 heterodimerization in vitro. In addition, two of the three Veli isoforms, Veli1 and -3 , are demonstrated to be expressed in the mouse retina. Immunofluorescence microscopy reveals extensive colocalization of Veli3 with both MPP4 and MPP5. This association of Veli3 with either MPP4 or MPP5 suggests that the MAGUKs recruit Veli3 and its binding partners to different cellular regions of the retina where they may participate in the organization of specialized intercellular junctions. J. Comp. Neurol. 481:31-41, $2005 . \quad \odot 2004$ Wiley-Liss, Inc.
\end{abstract}

Indexing terms: MPP4; MPP5; antibody; Veli isoforms; retina; intercellular junction

Scaffolding proteins are known to play a pivotal role in the organization of several types of cell-cell junctions, including interneuronal synapses and adhesive junctions of epithelial cells. Among the various classes of proteins involved in the formation and maintenance of junctional complexes, the membrane-associated guanylate kinase (MAGUK) family of proteins has emerged as important cytoplasmic adapters that support intercellular contacts by linking integral membrane proteins with cytoskeletal elements (e.g., Fanning and Anderson 1999; Gonzales-Mariscal et al., 2000; Roh and Margolis, 2003). The interactions are mediated by multiple protein-protein binding modules including the CaM kinase-like domain, the L27 heterodimerization domain, the PDZ domain, the SH3 mo- tif, the HOOK region, and the guanylate kinase-like (GUK) region (Dimitratos et al., 1997; Harris et al., 2002).

Grant sponsor: Deutsche Forschungsgemeinschaft; Grant number: STO 366/2-2; Grant number: WE 1259/14-2; Grant sponsor: Bundesministerium für Bildung und Forschung; Grant number: 01KW9921.

*Correspondence to: Heidi Stöhr, Institut für Humangenetik, Biozentrum, Am Hubland, Universität Würzburg, 97074 Würzburg, Germany. E-mail: heidi.stoehr@biozentrum.uni-wuerzburg.de

Received 29 March 2004; Revised 3 August 2004; Accepted 20 August 2004

DOI 10.1002/cne.20367

Published online in Wiley InterScience (www.interscience.wiley.com). 
Recent studies from us and other groups have provided evidence that two proteins of the p55-subfamily of MAGUKs exert important scaffolding function in the vertebrate retina. One of these, the gene encoding the membrane protein palmitoylated 4 (MPP4), was shown to be preferentially transcribed in human and mouse retina (Stöhr and Weber, 2000; Li et al., 2003). Subsequent immunohistochemical analyses of bovine and porcine retina showed that the MPP4 protein primarily localizes to the connecting cilia and the synaptic terminals of cone and rod photoreceptors in the outer plexiform layer (OPL) (Stöhr et al., 2003) and suggested a dual role of MPP4 in structural and functional distinct compartments of mammalian photoreceptors. Weaker labeling was also observed in postsynaptic structures of the OPL as well as in a subset of synapses in the inner plexiform layer (IPL) (Stöhr et al., 2003). In contrast, by examining mouse retinal sections, an exclusive localization of the MPP4 protein at rod photoreceptor spherules was reported (Li et al., 2003).

The second p55-like MAGUK, MPP5, with a proposed relevance to retinal function, was originally identified as the protein associated with mammalian homologs of Caenorhabditis elegans lin-7, 1 (Pals1) by bacterial expression cloning (Kamberov et al., 2000). It has been known for some time that the ortholog of MPP5 in Drosophila, stardust (Sdt), together with Crumbs (Crb) and Pals1associated tight junction (Patj), a PDZ protein formerly known as Discs-lost (Pielage et al., 2003), exist in a protein complex that regulates apico-basal polarity and contributes to the assembly of adherens junctions in epithelial cells (Bhat et al., 1999; Bachmann et al., 2001; Hong et al., 2001a). More recently, a ternary complex consisting of MPP5 and the Patj and Crb homologs, PATJ and CRB3, was identified at the tight junctions of mammalian epithelial cells (Lemmers et al., 2002; Roh et al., 2002; Makarova et al., 2003), indicating strong evolutionary conservation of this complex. Clues as to a possible role of the components of this complex in visual processes came from the identification of mutations in the gene of one of the Crb homologs in humans, CRB1, in patients with severe forms of retinitis pigmentosa (RP12) and Leber congenital amaurosis (den Hollander et al., 1999; Lotery et al., 2001). Furthermore, the analysis of Drosophila and mouse mutants of $\mathrm{Crb} / \mathrm{CRB} 1$ revealed that the proteins in both species are required for the expansion of the apical photosensitive membranes and the integrity of adherens junctions during photoreceptor development (Izaddoost et al., 2002; Pellikka et al., 2002; Mehalow et al., 2003). Interestingly, disruption of Crb or Sdt results in similar adult eye phenotypes in Drosophila, namely, shortened rhabdomeres and fragmentation of adherens junctions, suggesting an involvement of the two proteins in common cellular processes (Nam and Choi, 2003). Knowledge of the role of MPP5 in the vertebrate retina is still rudimentary and is mainly confined to the observation that zebrafish mutants with two larval-lethal recessive loss of function mutations in the MPP5/Std-ortholog nagie oko (nok) display severe defects in the cellular patterning of the retina (Wei and Malicki, 2002). Immunohistochemical analyses of zebrafish and mouse retina have localized nok/MPP5 in the vicinity of the outer limiting membrane (OLM) (Wei and Malicki, 2002; Mehalow et al., 2003). The OLM comprises a planar array of unique types of heterotypic adherens junctions formed between the photoreceptor and the surrounding Müller glial cells and between individual Müller cells (Pfaffenholz et al., 1999). In addition, mouse MPP5 was also reported to localize to synaptic layers of the retina (Mehalow et al., 2003), implying that MPP5 is involved in functional aspects of synaptic transmission.

MPP5 and other members of the p55 subfamily of MAGUKs (MPP2, MPP3, MPP6, and CASK) have been shown to bind mammalian homologs of C. elegans lin-7 via their C-terminal L27 domain (L27C) (Butz et al., 1998; Kamberov et al., 2000; Tseng et al., 2001). Three homologs of C. elegans lin-7 are known in mammals and are referred to as Veli1, -2, and -3 (Butz et al., 1998). In C. elegans, lin-7, lin-2 and lin-10 form a heterotrimeric complex that mediates the localization of the tyrosine kinase receptor Let-23 to the basolateral membranes of vulval epithelial cells (Kaech et al., 1998). A similar complex consisting of Veli and the mammalian orthologs of lin-2 (CASK) and lin-10 (Mint1/X11a) has been isolated from brain synapses (Butz et al., 1998), while in epithelial cells, CASK and Veli form a dimeric complex at basolateral membranes (Straight et al., 2000). Here, we analyzed MPP4 binding to Veli isoforms in vitro and, in addition, assessed a possible colocalization and physical association between MPP4 and MPP5 with the Veli proteins in membrane specializations of the retina.

\section{MATERIALS AND METHODS DNA constructs}

Constructs for bacterial expression of glutathione-Stransferase (GST) and maltose binding protein (MBP)fusion proteins were prepared by RT-PCR from bovine (MPP4) or mouse (MPP5) retinal RNA and introduced into the pGEX4T (Amersham Biosciences, Piscataway, NJ) or pMAL-c2 (New England Biolabs, Beverly, MA) vectors. Constructs contained the following amino acid (aa) residues: GST-MPP4-L27N (aa 1-89), GST-MPP4-L27C (aa 86-152), GST-MPP4-L27N+C (aa 1-152), GST-MPP4L27C+PDZ (aa 92-218), GST-MPP4-PDZ (aa 151-253), MBP-MPP4-L27C+PDZ (aa 92-218), GST-MPP5-N-term (aa 5-122), and MBP-MPP5-N-term (aa 5-122). GST and MBP fusion proteins were purified by glutathione sepharose beads (Amersham Biosciences) or amylose resins (New England Biolabs). The mammalian expression construct of bovine MPP4 in pCEP4.1 (Invitrogen, La Jolla, CA) has been previously described (Stöhr et al., 2003). The full length cDNA of human MPP5 was isolated by PCR with primers 5'-GAA TCA AGA GAA TTG GCT TAT AGG A-3' and 5'-CTC AGC CAA GTG GAT GGT A-3' using the cDNA clone DKFZp451E015 (obtained from RZPD, Berlin; GenBank acc. no. AL832326) as a template. Full-length Veli1, Veli2, and Veli3 cDNAs were generated by RT-PCR from mouse brain RNA using the following primer pairs: Veli1: 5'-GTT GCT GAT GCT GAA GCC GA-3' and 5'TGA CAT GTG GTT TTG TTG TG-3'; Veli2: 5' -CAT GGC TGC GCT GGT GGA GC-3' and 5'-ACC TCG AGA CTC CAA GGA CG-3'; Veli3: 5'-AGA AGA TGGC GGC CCT GGG-3' and 5'-GGT CTG TTG CCT GCG TTT TG-3'. The $2,093 \mathrm{bp}$ MPP5 cDNA fragment was inserted into the XhoI-BamHI site, the 706 bp Veli1 and 622 bp Veli2 cDNA fragments into the KpnI-HindIII site, and the $596 \mathrm{bp}$ Veli3 cDNA fragment into the HindIII site of the $\mathrm{pCEP} 4.1$ vector in-frame with a C-terminal rhodopsin (Rho)-1D4 tag (TETSQVAPA) (MacKenzie et al., 1984). All constructs were verified by direct sequencing. 


\section{Tissue source and protein extractions}

C57Bl/6 mice were purchased from Charles River Germany. Animals at postnatal day 1 (P1), P3, P5, and P7 were sacrificed by decapitation, and animals at P9, P11, $\mathrm{P} 15$, and adult mice were sacrificed by $\mathrm{CO}_{2}$ inhalation before their eyes were enucleated and the organs removed. All animal procedures were performed in accordance with the NIH guidelines for the use of animals in research and were approved by the Würzburg University Animal Care Committee. Fresh porcine and bovine eyes were obtained from the local slaughterhouse; human donor eyes were provided by the Würzburg University eye clinic in accordance to guidelines provided by the ethics committee of Würzburg University and the Declaration of Helsinki. Eyes from two to three mice from different litters at each developmental stage, the retinas and organs from two adult mice, and the retinas from a human, pig, and cow eye were used for protein extraction as described previously (Stöhr et al., 2003).

\section{Antibodies}

Rabbit peptide antibodies against MPP4 (MPP4-EP119) have been previously characterized (Stöhr et al., 2003). A polyclonal anti-MPP5 antiserum (MPP5-pAb) was generated in rabbits immunized with GST-MPP5-N-term fusion protein (ImmunoGlobe Antikörpertechnik) and purified using HiTrap NHS-activated sepharose HP columns (Amersham Biosciences). Briefly, the serum was first cleared of GST-specific antibodies by passing it through a GST column. Anti-MPP5-specific antibodies were then affinitypurified with a GST-MPP5-N-term column and used at 1:5,000 dilution for Western blotting (WB) and immunofluorescence (IF). For anti-MPP4 and anti-MPP5 monoclonal antibody (mAb) production, mice were immunized with an MBP-MPP4-L27C+PDZ or a GST-MPP5-N-term fusion protein-adjuvant mix (ImmunEasy Mouse Adjuvant, Qiagen, Chatsworth, CA), respectively. The spleens of two mice showing strong immune responses were used for cell fusion with NS-1 myeloma cells. Hybridomas secreting specific antibodies against MPP4 (3H10) and MPP5 (8H4) were cloned by limiting dilution. Hybridoma culture fluid of MPP5-8H4 was used for WB and IF. MPP4-3H10 was either used as tissue culture supernatant (WB, IF) or as purified $\mathrm{mAb}$ at a concentration of 8 $\mu \mathrm{g} / \mathrm{ml}$ (IF). Other antibodies in this study were the $\mathrm{mAb}$ against rhodopsin (clone 1D4; MacKenzie et al., 1984) (WB, 1:40), pan-cadherin (clone CH-19; Sigma, St. Louis, MO) (IF, 1:500), and glutamine synthetase (Chemicon, Temecula, CA) (IF, 1:1,000), the polyclonal goat antibody directed against Veli1 (Santa Cruz Biotechnology, Santa Cruz, CA) (WB, 1:300) and polyclonal rabbit antibodies against Veli3 (WB, IF, 1:200) and ZO-1 (IF, 1:50) (both Zymed, San Francisco, CA). Secondary antibodies included immunoglobulins G (IgGs) conjugated to horseradish peroxidase (Calbiochem, La Jolla, CA) (WB, 1:5,000) and IgGs conjugated to Alexa 488 or Alexa 594 (Molecular Probes, Eugene, OR) (IF, 1:1,000).

\section{Adsorption of antibodies with recombinant proteins}

To evaluate the specificity of the MPP4 and MPP5 antibodies, $100 \mu \mathrm{l}$ of tissue culture supernatant or diluted purified antibodies were preadsorbed for 4 hours at $4^{\circ} \mathrm{C}$ with $200 \mu \mathrm{g}$ of either GST-MPP4-L27C+PDZ or GST-
MPP5-N-term fusion protein immobilized on glutathione sepharose beads. The beads were pelleted at $1,000 \mathrm{~g}$ for 3 minutes and the supernatants were tested on cryosections by immunofluorescence.

\section{Immunofluorescence labeling}

For immunohistochemistry, the corneas of excised eyes from adult mice were perforated and the entire eyecups were immersion-fixed in freshly prepared $4 \%$ paraformaldehyde in $0.1 \mathrm{M}$ phosphate buffer (PB, $\mathrm{pH} 7.4$ ) for 1 hour at room temperature. After rinsing several times in $0.1 \mathrm{M}$ $\mathrm{PB}$, the eyes were cryoprotected in $0.1 \mathrm{M}$ PB containing $18 \%$ sucrose for 12 hours. The eyecups were then embedded in OCT embedding medium (Tissue-Tek), fast-frozen in liquid nitrogen, and cryosectioned vertically at $10 \mu \mathrm{m}$. Sections were stored at $-80^{\circ} \mathrm{C}$ until use. Paraformaldehyde fixation of porcine retinas was performed as described previously (Stöhr et al., 2003). After thawing the cryosections were blocked with $0.1 \mathrm{M}$ PB containing $0.3 \%$ Triton X-100 and $10 \%$ goat serum for 30 minutes. Labeling with hybridoma culture supernatant or purified antibodies diluted in $0.1 \mathrm{M} \mathrm{PB}, 0.1 \%$ Triton $\mathrm{X}-100$, and $2.5 \%$ goat serum was performed overnight at room temperature. After washing in 0.1 M PB, the sections were incubated with secondary antibodies for 1 hour at room temperature. Double-labeling studies were performed by simultaneously applying the primary antibodies followed by a mixture of secondary antibodies. Each immunolabeling experiment was performed on eye sections from at least two animals. Labeled sections were washed in $0.1 \mathrm{M}$ PB and mounted (Confocal Matrix, Micro-Tech-Lab). Labeled specimen were examined under an Axioskop-2 mot plus fluorescence microscope equipped with appropriate fluorescent filters (Zeiss). Images were captured with a digital AxioCam MRc camera (Zeiss), processing and storage were achieved with the AxioVision software v. 3.1 with integrated Z-stack, 3D deconvolution, and extended focus modules (Zeiss). Brightness and contrast were adjusted.

\section{Isolation of single cells}

Müller glial cells were freshly isolated from retinas of adult mice as described previously (Biedermann et al., 2002). Briefly, the retinas were incubated in $\mathrm{Ca}^{2+}$ - and $\mathrm{Mg}^{2+}$-free PBS containing papain $(0.1 \mathrm{mg} / \mathrm{ml})$ for $30 \mathrm{~min}$ utes at $37^{\circ} \mathrm{C}$ followed by a wash in PBS with $200 \mathrm{U} / \mathrm{ml}$ DNase I (Sigma). After fixation with $4 \%$ paraformaldehyde at $4^{\circ} \mathrm{C}$ for 10 minutes, the retinas were washed in PBS and the tissue pieces were gently triturated by a wide-pore pipette to obtain suspensions of isolated cells. For immunofluorescence staining the cells were transferred to gelatin-coated slides, air-dried, and immediately labeled as described above.

\section{Real-time quantitative RT-PCR}

Total RNA isolated from mouse retina, brain, heart, skeletal muscle, kidney, liver, and lung was used to synthesize first-strand cDNA by standard procedures (Stöhr et al., 2001). Real-time quantitative reverse transcriptasePCR (qRT-PCR) and normalization of gene expression was performed as described (Krämer et al., 2004). Primer pairs for qRT-PCR analysis were as follows: Veli1, 5' -CAA CAG CAG CAG CAA CAA-3' and 5'-CTT TCC TTC GAG CGC CTA T-3'; Veli2, 5' -TCA GTG AAA CTG GTG GTG-3' and 5'-GAG ACT CCA AGG ACG TGT A-3'; Veli3, 5'-CTC 
$\mathbf{A}$

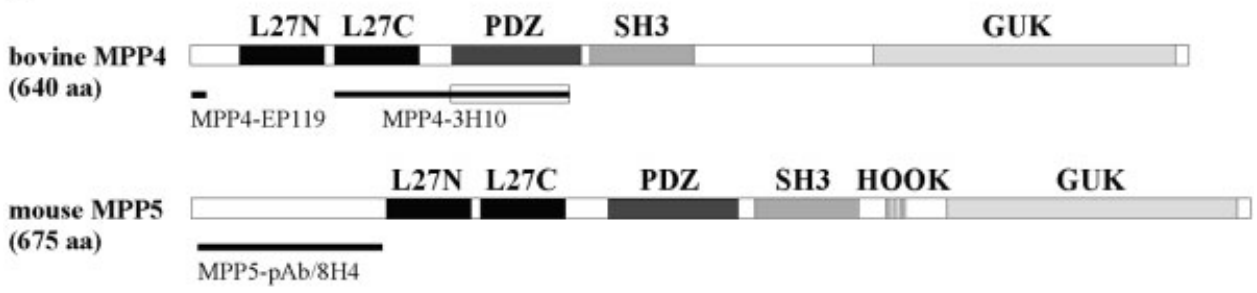

B

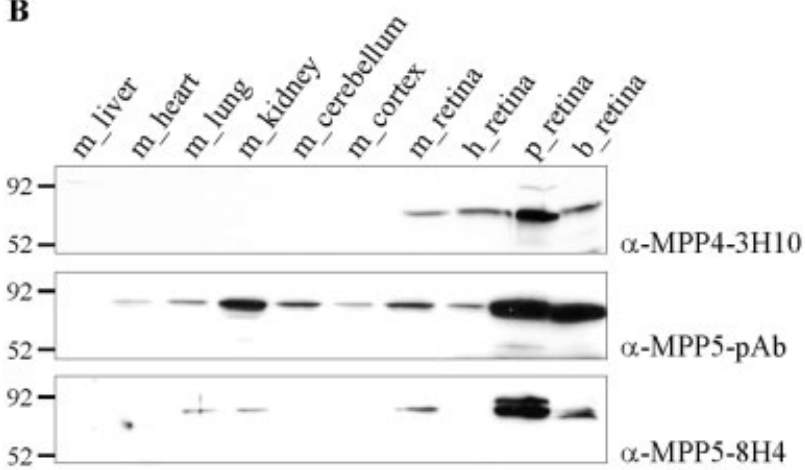

C

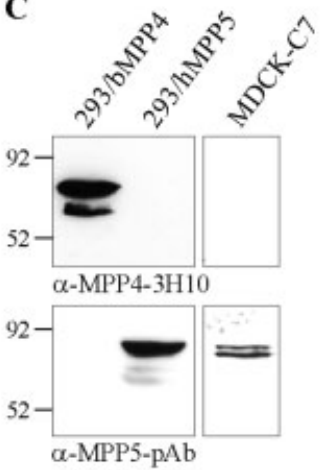

Fig. 1. Design and characterization of anti-MPP4 and anti-MPP5 antibodies. A: Schematic of bovine MPP4 and mouse MPP5 illustrating the protein interaction domains by shaded boxes. The regions selected as antigens for antibody production are indicated below the respective proteins. The open box denotes the refined region of epitope recognition for monoclonal antibody MPP4-3H10. B: Western blot analysis of mouse tissues and various mammalian retinal homogenates probed with the cell culture supernatants of hybridoma cell lines MPP4-3H10, MPP5-8H4, and affinity-purified polyclonal rabbit MPP5 antiserum (MPP5-pAb). Note that the signals produced by MPP5-pAb in bovine and porcine retina correspond to two bands. A second slightly lower band was also observed in the other tissues after prolonged exposure (data not shown). C: Lysates of 293-EBNA cells overexpressing bovine MPP4 or human MPP5, and MDCK-C7 cells were immunoblotted with MPP4-3H10 and MPP5-pAb antibodies.
AAA CGA GGA GAT CAG CTA-3' and 5'-ATT AGG TCT GTT GTC TGC GTT-3'. All samples were analyzed in triplicate.

\section{Cell transfection and GST pulldown assays}

293-EBNA cells (Invitrogen) were maintained in

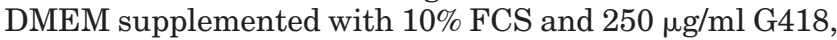
MDCK-C7 cells (kindly supplied by Dr. M. Gekle, University of Würzburg) in MEM supplemented with 10\% FCS. Transient transfections were performed as described previously (Stöhr et al., 2003). A stable 293-EBNA cell line expressing MPP4 was generated by selection of hygromycin B resistant colonies. Transfected cells were solubilized in TBS (20 mM Tris pH 7.4, 0.15 M NaCl)/1\% Triton X-100 supplemented with complete protease inhibitor cocktail tablets (Roche Applied Sciences, Nutley, NJ) for $30 \mathrm{~min}-$ utes and centrifuged at $16,000 \mathrm{~g}$ for 15 minutes at $4^{\circ} \mathrm{C}$. For GST pulldown assays, transfected 293-EBNA cell lysates were incubated with GST (control) or GST fusion proteins immobilized on glutathione-sepharose beads for 1 hour at $4^{\circ} \mathrm{C}$. After several washes with TBS $/ 0.1 \%$ Triton $\mathrm{X}-100$, bound proteins were eluted with $2 \times$ Lämmli loading buffer and immunoblotted as described previously (Stöhr et al., 2003).

\section{Immunoprecipitations}

Porcine retinas were homogenized in 10 volumes $(\mathrm{w} / \mathrm{v})$ of TBS supplemented with protease inhibitor cocktail and centrifuged at $100,000 \mathrm{~g}$ for 15 minutes at $4^{\circ} \mathrm{C}$ to yield a crude membrane fraction. Retinal membranes were then solubilized in TBS containing 1\% deoxycholate for $30 \mathrm{~min}-$ utes, Triton-X 100 was added to $1 \%$, and the extract was cleared by centrifugation at $100,000 \mathrm{~g}$ for 30 minutes. Affinity-purified primary antibodies $(2 \mu \mathrm{g})$ or $5 \mu \mathrm{l}$ of rabbit serum were added to aliquots of solubilized retinal membranes or Triton X-100-solubilized transfected 293-EBNA cell lysates and incubated for 4 hours at $4^{\circ} \mathrm{C}$. To precipitate antibodies, $40 \mu \mathrm{l}$ of protein A-sepharose (50\% slurry in TBS $/ 0.1 \%$ Triton-X 100) (Sigma) was added and the samples were incubated for 4 hours at $4^{\circ} \mathrm{C}$. After three washes with TBS/0.1\% Triton-X 100, the immunoprecipitates were boiled in $2 \times$ Lämmli loading buffer and analyzed by Western blotting.

\section{RESULTS}

\section{Mono- and polyclonal antibodies against MPP4 and MPP5}

MPP4 and MPP5 proteins share an overall sequence identity of $33 \%$ and a similarity of $52 \%$, while displaying a similar domain architecture with two tandem L27 heterodimerization modules, $\mathrm{L} 27 \mathrm{~N}$ and $\mathrm{L} 27 \mathrm{C}$, and the characteristic MAGUK tripartite core region consisting of a PDZ, an SH3 and a GUK domain (Fig. 1A). MPP5 differs from MPP4 by a longer N-terminal extension of $\sim 100$ amino acids and a HOOK region with a protein 4.1 binding site (Fig. 1A). A peptide antibody generated against 
the extreme N-terminus of bovine MPP4 (MPP4-EP119) was shown to specifically label the MPP4 protein in bovine retinal extracts as well as in bovine and porcine retinal sections (Stöhr et al., 2003). MPP4-EP119 does not crossreact with MPP4 in other mammalian species, probably due to the low interspecies conservation of the $\mathrm{N}$-terminus. We have now generated a monoclonal antibody against a more central region of bovine MPP4 (MPP4-3H10) (Fig. 1A). By immunoblotting of fusion proteins containing different portions of MPP4, the epitope of MPP4-3H10 was positioned within the PDZ domain (data not shown). On Western blots containing total protein samples from different mouse tissues as well as porcine, human, and bovine retina, MPP4-3H10 specifically detected the $\sim 70 \mathrm{kDa}$ MPP4 protein in retinal extracts from all species but not in mouse liver, heart, lung, kidney, cerebellum, and cortex (Fig. 1B). This provides further confirmation that MPP4 is a retina-specific protein in mammals.

Probing of multiple mouse tissue Western blots with poly- and monoclonal antibodies raised against the $\mathrm{N}$-terminal region of mouse MPP5 demonstrated the presence of the MPP5 protein in various mouse tissues. In particular, both MPP5 antibodies readily detected a protein migrating at $\sim 80 \mathrm{kDa}$, which corresponds to the calculated molecular weight of MPP5, in mouse lung, kidney, and retina (Fig. 1B). In addition, the polyclonal antibody (MPP5-pAb) recognized significant amounts of MPP5 protein in heart, cerebellum, and cortex. Faint bands in these tissues were also produced by the monoclonal MPP5 antibody (MPP5-8H4) after long exposure (data not shown). Our results are in good accordance with Northern blot analyses revealing a broad tissue distribution of the mouse Mpp5 mRNA with highest expression in kidney and placenta (Kamberov et al., 2000). Interestingly, whereas both antibodies crossreacted with MPP5 from bovine and porcine retina, MPP5 in human retina was only detected by MPP5-pAb (Fig. 1B).

To further test the specificity of the MPP4 and MPP5 antibodies, cell lysates of 293-EBNA overexpressing bovine MPP4 or human MPP5 were analyzed by Western blotting. MPP4-3H10 specifically identified the MPP4 protein, while MPP5-pAb exclusively labeled the MPP5 protein (Fig. 1C). In epithelial MDCK-C7 cells, endogenous MPP5 was detected as a doublet when immunoblotted with MPP5-pAb (Fig. 1C). Interestingly, immunoprecipitated endogenous MPP5 from MDCK cells has previously been reported to be recognized as two bands on Western blots (Makarova et al., 2003).

\section{MPP4 and MPP5 proteins in the mouse retina}

Immunofluorescence labeling of frozen mouse retinal sections with MPP4-3H10 hybridoma culture fluid repeatedly produced intense labeling of the photoreceptor terminals in the OPL. When purified MPP4-3H10 was used as the primary antibody additional, weaker punctate and elongated staining of structures at the distal ends of the photoreceptor inner segments was observed (Fig. 2A). Preadsorption of MPP4-3H10 with GST-MPP4L27C+PDZ fusion protein completely abolished the immunostaining in the OPL (Fig. 2A). The MPP4-3H10derived immunoreactivity at the junction between photoreceptor inner and outer segments, however, could not be blocked by preincubating the purified MPP4-3H10 antibody with recombinant fusion protein (Fig. 2A).

Both, mono- and polyclonal antibodies against MPP5 brightly and exclusively stained the OLM region of mouse retina, thus providing strong evidence for the specificity of the immunolabeling (Fig. 2B). Additional control experiments showed that preadsorption of the MPP5-pAb with excess GST-MPP5-N-term fusion protein entirely eliminated the OLM staining (Fig. 2B). The horizontal staining of the OLM produced by the MPP5 antibodies appeared to be noncontinuous and had a weaving pattern appearance. To further define the localization of MPP5 at the intercellular junctions of the OLM region, we first performed double-labeling on mouse retinal sections using MPP5$\mathrm{pAb}$ in conjunction with monoclonal antibodies against classical cadherins and glutamine synthetase (GS). Cadherins function as adhesion molecules and have repeatedly been localized to the adherens junctions formed between the photoreceptor IS and Müller cells in the OLM (Pfaffenholz et al., 1999). GS is an enzyme that is exclusively expressed in the Müller glial cells of the retina (Linser et al., 1984). Our data indicate that MPP5 is positioned apical to the adherens junctions labeled by the pan-cadherin antibody (Fig. 2C). They also show that most of the MPP5 stained structures overlapped with the apical membranes of Müller cells labeled by the GS antibody (Fig. 2D). MPP5 immunoreactivity was clearly absent from the fine GS-positive threads representing the microvillous processes of Müller cells projecting into the subretinal space (Fig. 2D). To further evaluate the presence of MPP5 protein in Müller glial cells, we performed immunocytochemical staining of isolated mouse Müller cells. Prominent MPP5 immunoreactivity was repeatedly found to decorate the distal region of the glial cells (Fig. $2 \mathrm{E})$. Here, the presence of MPP5 coincides with the tight junction protein ZO-1 (Fig. 2E), a known component of mouse OLM junctions (Tserentsoodol et al., 1998).

\section{Isoform-specific expression of Veli genes in mouse retina}

In a first step to study complex formation of MPP4 and MPP5 with Veli proteins in the retina, we sought to accurately determine the gene expression profile of each Veli isoform in a panel of seven adult mouse tissues including retina, brain, heart, skeletal muscle, kidney, lung, and liver by sensitive real-time qRT-PCR analysis (Fig. 3). Primer pairs were designed to unambiguously amplify isoform-specific mRNAs. This was confirmed by direct sequencing of the RT-PCR products. Veli1, which has previously been reported to be a brain-specific gene in rat (Jo et al., 1999), was shown to be expressed at varying levels in all mouse tissues tested. Veli3, a ubiquitously expressed gene in rat (Jo et al., 1999) showed similar levels of expression in the different mouse tissues. Concordant with the Northern blot analyses performed in rat (Jo et al., 1999), Veli2 mRNA was abundantly present in brain with lower levels of Veli2 expression found in heart and skeletal muscle. No Veli2 transcripts were detected in mouse retina (Fig. 3). Thus, based on the high expression of Veli1 and Veli3 in the mouse retina, these proteins were regarded as candidates for retinal binding partners of MPP4 and MPP5. 
A

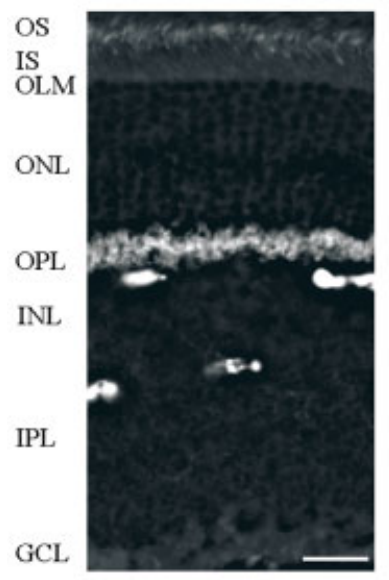

MPP4-3H10

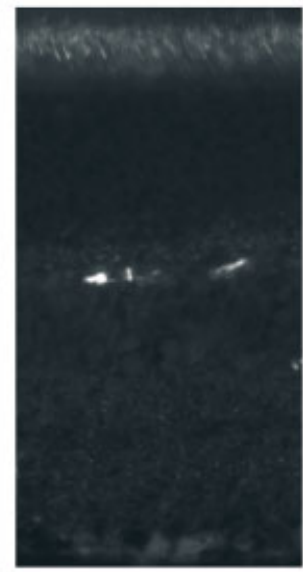

MPP4-3H10 blocked
B

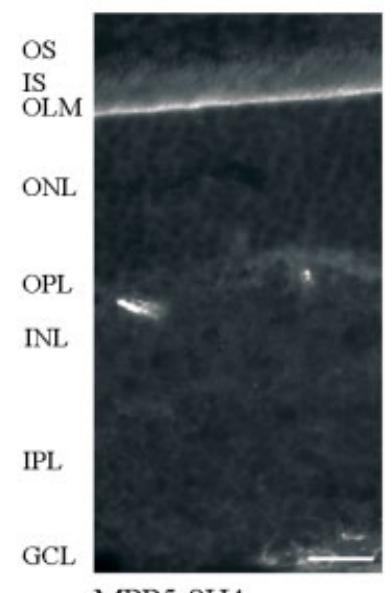

MPP5-8H4

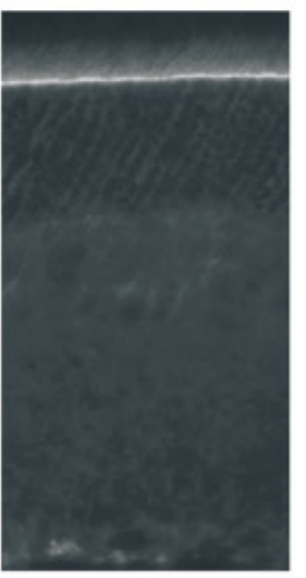

MPP5-pAb

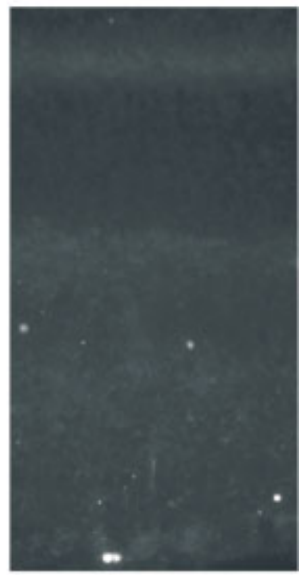

MPP5-pAb blocked
C

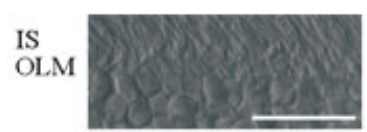

D

DIC

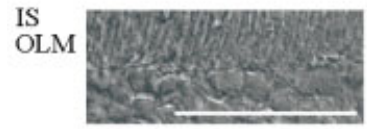

DIC

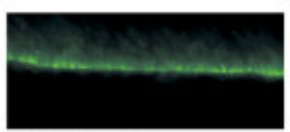

MPP5-pAb

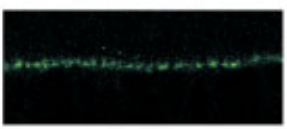

MPP5-pAb

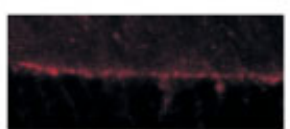

pan-cadherin
E

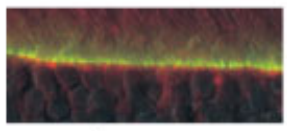

merged

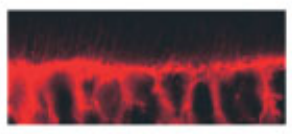

GS

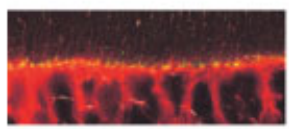

merged

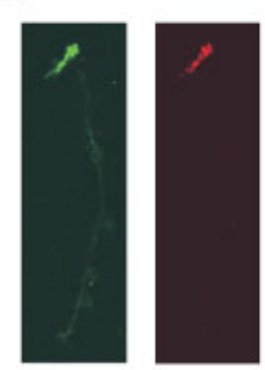

MPP5-8H4 ZO-1

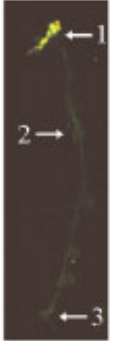

merged
Fig. 2. Cellular localization of MPP4 and MPP5 in the mouse retina. A: Immunofluorescence microscopy on cryostat sections of mouse retina labeled with MPP4-3H10 and MPP4-3H10 preadsorbed with MPP4 fusion protein. B: Immunolabeling of retinal sections with MPP5-8H4, MPP5-pAb, and MPP5-pAb antibodies preincubated with MPP5 fusion protein. Note that endogenous mouse IgG containing blood vessels in the inner retina were stained by the fluorescein conjugated secondary anti-mouse IgG antibodies when mouse primary antibodies MPP4-3H10 and MPP5-8H4 were used. C,D: Outer retina simultaneously stained with the MPP5-pAb (green) and pan- cadherin (C) or glutamine synthetase (GS) (D) antibodies (both red). Immunofluorescence micrographs were merged with the respective differential interference contrast (DIC) image. Scale bars $=25 \mu \mathrm{m}$. OS, outer segments; IS, inner segments; OLM, outer limiting membrane; ONL, outer nuclear layer; OPL, outer plexiform layer; INL, inner nuclear layer; IPL, inner plexiform layer; GCL, ganglion cell layer. E: Immunocytochemical staining of a single Müller glial cell with MPP5-8H4 and ZO-1 antibodies. The merged image is shown on the right. The arrows indicate the distal end (1), the soma (2), and the endfoot (3) of the Müller cell. Yellow indicates colocalization.

\section{MPP4 binds Veli isoforms via the $\mathrm{L27C}$ heterodimerization domain}

Veli1, -2, and -3 share over $80 \%$ peptide sequence identity complicating the generation of antibodies for distinct isoforms. We therefore analyzed the specificity of commercially available anti-Veli1 and anti-Veli3 antibodies by overexpressing the three Velis as Rho-1D4-tagged proteins in 293-EBNA cells. Immunoblotting of the cell lysates with Rho-1D4 antibodies showed that each of the Veli proteins migrated at a mobility that corresponds to the reported molecular masses of the Veli isoforms, Veli1 at $\sim 30 \mathrm{kDa}$ and both Veli2 and Veli3 at $\sim 25 \mathrm{kDa}$ (Jo et al., 1999; Fig. 4A). Furthermore, antibodies directed against Veli1 or Veli3 specifically recognized their target protein in 293-EBNA cell transfected with Rho-1D4-tagged Veli1 or Veli3, respectively, and showed no crossreactivity with each other or Veli2 (Fig. 4A). Note that Veli3 is endogenously expressed in 293-EBNA cells. Both Veli1 and
Veli3 antibodies detected the respective endogenous isoform in mouse retinal extracts.

We next coexpressed MPP4 and Veli1 or Veli3 in 293EBNA cells and performed coimmunoprecipitations with the cell lysates to test for interaction. Western blotting of precipitated proteins showed that Veli1 and Veli3 were coimmunoprecipitated by the MPP4-EP119 antibody and, conversely, both antibodies against Veli1 and Veli3 were able to coimmunoprecipitate MPP4 (Fig. 4B). GST proteins fused to the L27N, the L27C or both L27 domains of MPP4 were used to analyze the binding of the overexpressed Rho-1D4-tagged Veli isoforms by GST pulldown assays. Only GST fusion proteins containing the L27C module bound Veli1, -2, or -3, and binding was found to be most efficient with the GST-L27N+C construct (Fig. 4C). These results indicate that MPP4 binds all three Veli isoforms in vitro and that the interaction is mediated by the L27C domain. 


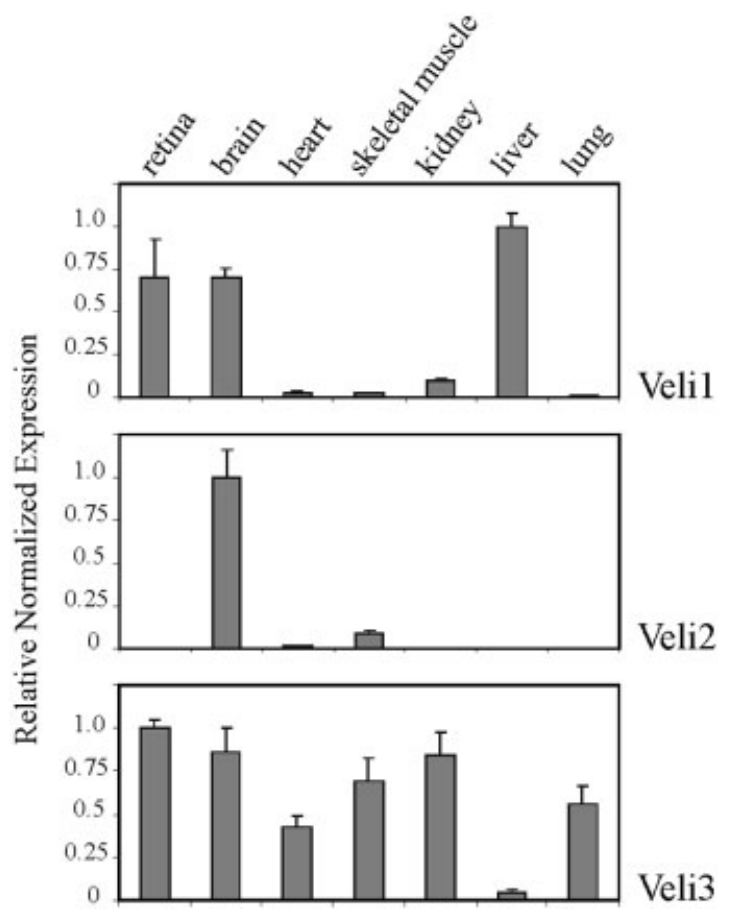

Fig. 3. RNA expression pattern of mouse Veli1, Veli2, and Veli3 obtained by real-time qRT-PCR analysis. The relative normalized expression values for each tissue are indicated by shaded bars and are given in logarithmic scale. Error bars denote standard deviations of reactions performed in triplicate.

\section{Colocalization of Veli3 with MPP4 and MPP5 in the retina}

In the mouse brain, the Veli isoforms were shown to be present in discrete neuronal populations whereby most neurons expressed only one of the three Velis (Misawa et al., 2001). To evaluate the cellular localization of Veli1 and Veli3 in the retina, we performed immunofluorescence labeling on mouse retinal cryosections using the available Veli antibodies. Several attempts to label mouse retina with the Veli1 antibody were unsuccessful, strongly suggesting that this antibody is not useful for immunohistochemistry.

The Veli3 antibody produced the most intense staining in photoreceptor terminals of the OPL (Fig. 5A). In the outer retina, Veli3 was also detected in the OLM region as well as in a subset of photoreceptor cell bodies and their axonal processes in the outer nuclear layer (ONL) (Fig. $5 \mathrm{~A}, \mathrm{~B})$. The arrangement of the cell bodies of Veli3immunopositive photoreceptor cells at the outermost portion of the retina, beneath the OLM, is reminiscent of the cone-specific labeling of antibodies against the neuronspecific enolase (Rich et al., 1997) and suggest that the Veli3 protein is preferentially expressed in mouse cone photoreceptors. Merging of the immunofluorescence micrographs obtained from double-labeling with Veli3 and MPP5-8H4 antibodies showed that the horizontal line of Veli3-stained structures in the OLM region overlaps with MPP5-immunoreactivity (Fig. 5B). In the OPL, doublelabeling with the Veli3 and MPP4-3H10 antibodies demonstrated that Veli3 extensively colocalizes with MPP4 at the photoreceptor synaptic membranes (Fig. 5C). Consistent with the colocalization patterns observed in mouse, double-labeling studies on porcine retinal sections revealed overlapping expression of Veli3 with MPP4 and MPP5 in the OPL and OLM, respectively (data not shown).

In the INL of the murine retina, the Veli3 antibody labeled numerous cell bodies of presumptive bipolar cells and their axonal extensions descending to the IPL (Fig. 5A). Moderate Veli3 immunoreactivity was also broadly distributed in synaptic terminals throughout the IPL (Fig. 5A). Immunohistochemical analysis of porcine retinas with the Veli3 antibody produced intense staining of the OLM, OPL, and IPL; the cell bodies of cone photoreceptors and interneurons, however, were not labeled in pig (data not shown).

\section{Expression of MPP4, MPP5, and Veli3 during retinal development}

To determine whether the MPP4, MPP5, and Veli3 proteins are likely to be involved in mouse retinogenesis, we evaluated their expression by Western blotting throughout postnatal retinal development. MPP4 protein was first detected at $\mathrm{P} 7$ and the expression progressively increased until the end of the second postnatal week (Fig. 6). This is consistent with the steep increase of Mpp4 mRNA expression from P5 as observed by real-time qRT-PCR analysis (Li et al., 2003) and coincides with the onset of ribbon synapse formation between photoreceptors and their secondary neurons in the OPL (Rich et al., 1997). In contrast to MPP4, MPP5 and Veli3 proteins were detected at a constant level throughout postnatal development of the mouse retina (Fig. 6).

\section{Analysis of in vivo binding between Veli3 and MPP4 and MPP5 in the retina}

The overlapping expression of Veli3 with MPP4 and MPP5 at distinct cellular regions of the retina suggests that Veli3 directly associates with either of these proteins in this tissue. To address this possibility, immunoprecipitations were performed on solubilized porcine retinal membranes (Fig. 7). Immunoblotting of the precipitates showed that the antibodies against Veli3, MPP4, and MPP5 specifically bound their target protein in retinal extracts. The analysis of MPP4 immunoprecipitates revealed that Veli3 is coimmunoprecipitated with the MPP4 antibody. Accordingly, MPP4 is copurified with the Veli3 antibody. Thus, a native complex containing Veli3 and MPP4 was demonstrated to be present in retina. Immunoblotting of Veli3 immunoprecipitates with the MPP5 antibody failed to detect retained MPP5 protein. Nevertheless, a faint signal was observed when MPP5 precipitates were probed with the Veli3 antibody, indicating that at least small amounts of complexes between MPP5 and Veli3 exist in the retina. The band migrating at about the height of the Veli3 protein in control serum precipitates represents the IgG light chains which are recognized by the secondary goat anti-rabbit IgG antibody.

\section{DISCUSSION}

The precise cellular localization of the p55-like MAGUK proteins MPP4 and MPP5 in the multilayered retina is an important step towards our understanding of their biolog- 

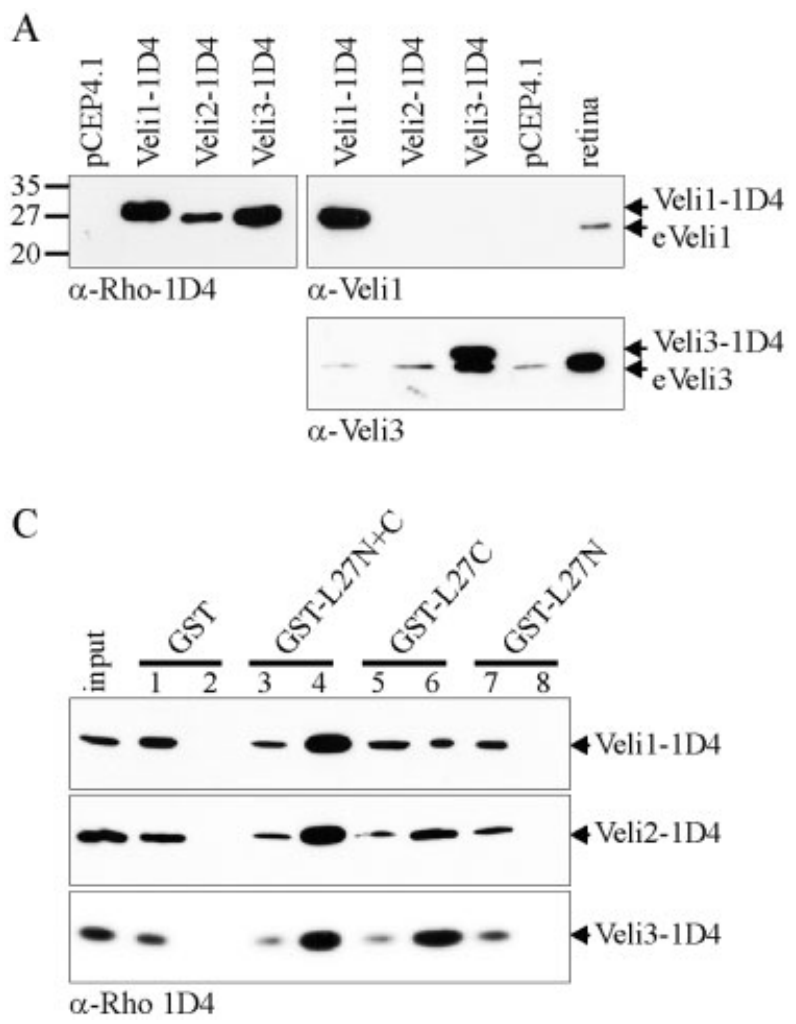

Fig. 4. Binding of MPP4 to Veli isoforms. A: The three Rho-1D4tagged Veli isoforms expressed in 293-EBNA cells were detected by immunoblotting with Rho-1D4. Specificity of isoform-directed antibodies against Veli1 and Veli3 is shown by immunoblotting of cell lysates with the overexpressed Rho-1D4-tagged Veli isoforms and mouse retinal extracts. Endogenous Veli1 and Veli3 (eVeli1, eVeli3) were identified in mouse retina; eVeli3 was also present in 293-EBNA cells (lower band). B: Proteins from lysates of 293-EBNA cells coexpressing MPP4 and Veli1 or Veli3 were immunoprecipitated (IP) with

ical role in this tissue and facilitates immunocytochemical colocalization studies to evaluate the in vivo significance of putative protein-protein associations. To further assess previously reported immunofluorescence analyses, we have now generated novel antibodies against both proteins.

In the present study, we demonstrate that a monoclonal antibody against bovine MPP4 specifically recognizes the MPP4 protein in retinal extracts from various mammalian species including mouse, cow, pig, and human. The antibody intensely labeled the synaptic terminals of photoreceptor cells in mouse and pig and thus confirmed the expression of MPP4 in the ribbon synapse (Stöhr et al., 2003; Li et al., 2003). Previously, the MPP4 protein was demonstrated to be specifically recognized by a rabbit peptide MPP4 antibody in the photoreceptor cilia and proximal inner retinal layers of cow and pig (Stöhr et al., 2003), but not by a peptide antibody in mouse ( $\mathrm{Li}$ et al., 2003). Immunoreactivity in inner retinal layers was not detected with the monoclonal antibody MPP4 and the specificity of the staining produced by purified monoclonal MPP4 antibodies at the distal ends of the mouse photoreceptor inner segments could not be validated by adsorption controls. Inconsistencies in the cellular localization of
B

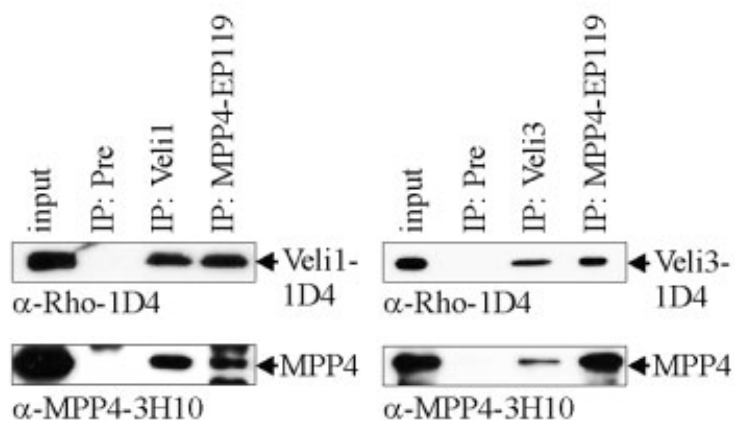

preimmune serum (Pre) as a control and MPP4-EP119, Veli1 or Veli3 antibodies. Bound proteins were immunoblotted with Rho-1D4 to detect Veli isoforms or MPP4-3H10 to detect the MPP4 protein. C: GST-pulldown assays. 293-EBNA cell lysates containing the overexpressed Rho-1D4-tagged Veli isoforms were bound to the indicated GST-fusion proteins and analyzed by immunoblotting with Rho-1D4. Lanes 1, 3, 5, 7: unbound fractions; lanes 2, 4, 6, 8: bound fractions. Input was $5 \%$ of total 293-EBNA cell lysate used for IP or GSTpulldowns.

other retinal proteins, in particular to the photoreceptor cilium, have been reported, e.g., the retinitis pigmentosa guanosine triphosphatase (GTPase) regulator (RPGR) (Yan et al., 1998; Hong et al., 2001b; Mavlyutov et al., 2002), and were mainly attributed to the unique anatomical characteristics of this subcellular compartment (Hong et al., 2003). The connecting cilium consists of a $9 \times 2+0$ microtubuli axoneme which is linked to large surface glycoconjugates by specific crosslinkers (Horst et al., 1990). In addition, it is ensheathed by a close meshed interphotoreceptor matrix (Tawara et al., 1988). It has been assumed that this material can block antibody penetration and antigen binding in the connecting cilium (Hong et al., 2003). The MPP4 antibodies are directed against different epitopes of the MPP4 protein, namely, the N-terminus (Stöhr et al., 2003), the PDZ domain (present study), and a region between the $\mathrm{SH} 3$ and GUK domain (Li et al., 2003). Thus, it is conceivable that the accessibility of different antigenic regions varies within the submembraneous compartments of the cilium. Immunofluorescence labeling in the present study was performed by a preembedding fixation procedure-other protocols to further perforate the interphotoreceptor matrix and unmask epitopes may be successful to consistently restore the 
A

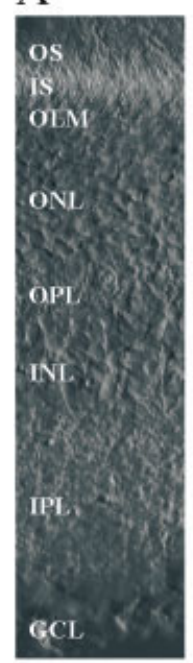

DIC
B

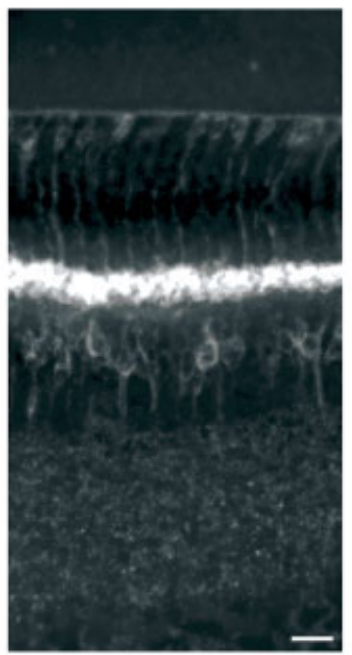

Veli3

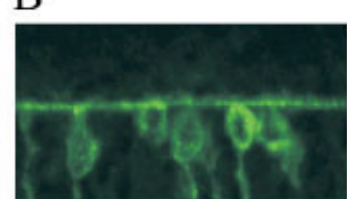

Veli3

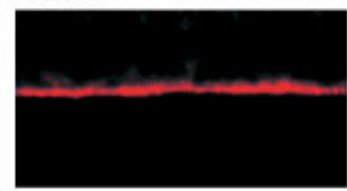

MPP5-8H4

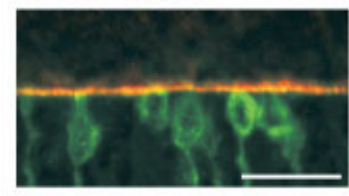

merged
C

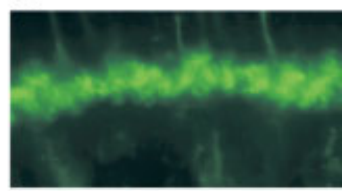

Veli3

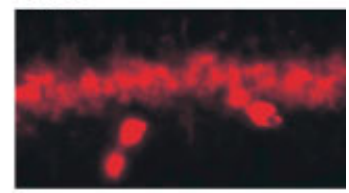

MPP4-3H10

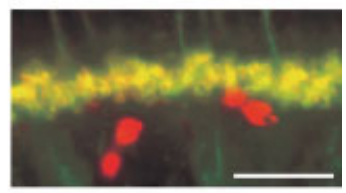

merged $10 \mu \mathrm{m}$

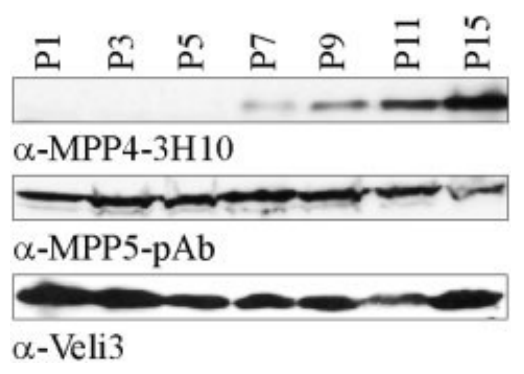

Fig. 6. Western blot analysis of MPP4, MPP5, and Veli3 protein expression during postnatal development of the mouse retina. Protein extracts from different developmental stages were immunoblotted with MPP4-3H10, MPP5-pAb, and Veli3 antibodies. The total amount of proteins loaded in each lane was adjusted to be equal.

MPP4 antigenicity for MPP4 antibodies in the connecting cilium. In addition, alternative techniques, such as serial retinal sectioning or the preparation of enriched ciliary axonemes, are most likely required to further resolve this issue.

Immunofluorescence labeling with different mono- and polyclonal antibodies directed against MPP5 consistently revealed strong MPP5 immunoreactivity at the outer limiting membrane specializations formed between the photoreceptor and Müller glial cells. Moreover, we observed MPP5 distal to cadherin-containing adherens junctions, a finding that coincides with the detection of the MPP5 ortholog nok apical to the OLM junctions in zebrafish (Wei and Malicki, 2002). Mouse CRB1, a putative interaction partner of MPP5 in the retina, was localized to radial and apical processes of Müller cells as well as the photoreceptor IS (Pelikka et al., 2002; Mehalow et al., 2003). Our data on retinal sections and isolated Müller cells strongly indicate MPP5 expression in Müller cells, where it colocalizes with glutamine synthetase and ZO-1 at the basis of the apical microvillous processes. In addition, MPP5 may also be present at opposing membranes of the photoreceptor inner segments.

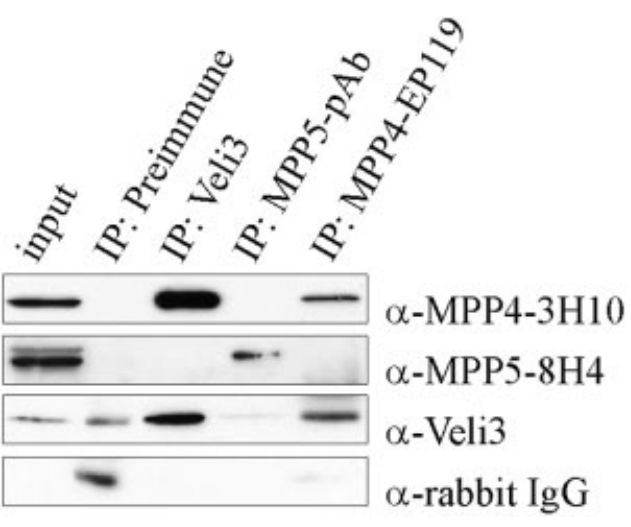

Fig. 7. Analysis of complex formation of MPP4 and MPP5 with Veli3 in the retina. Solubilized porcine retinal membranes were immunoprecipitated with rabbit preimmune serum and rabbit antibodies against Veli3, MPP5, and MPP4. Precipitates were analyzed by immunoblotting with the antibodies shown on the right. Input was 5\% of total retinal membranes. The bands detected with the Veli3 and goat anti-rabbit IgG antibodies in the lane containing proteins bound by the preimmune serum correspond to the IgG light chains.

Bright staining of synaptic layers with a polyclonal antibody against the C-terminus of mouse MPP5 has been reported; however, the information on the precise location, namely, outer versus inner plexiform layers, is ambiguous (Mehalow et al., 2003). Unfortunately, the specificity of the MPP5 antibody in this study was not demonstrated. Both, our mono- and polyclonal MPP5 antibodies failed to label synaptic terminals in mouse retina, while immunoblotting and immunohistochemistry validated the specificity of these MPP5 antibodies. Antibody-dependent variation in the immunolocalization of the MPP5 protein may be due to variation in fixation or embedding protocols. While we used cryosections for immunolabeling, paraffinembedded eye sections were immunostained by Mehalow et al. (2003). Nevertheless, supported by the fact that 
protein products of the MPP5 orthologs in zebrafish (nok) and Drosophila (Sdt) solely distribute to positionally analogous regions in the fish and fly eye (Wei and Malicki, 2002; Nam and Choi, 2003), our results strongly argue for an exclusive localization of MPP5 in membrane specializations distal to the adherens junctions of the OLM in the mammalian retina.

The p55-like MAGUK proteins MPP4 and MPP5 possess several conserved protein interaction domains that are likely involved in the formation of extensive protein networks. An important and often unclear aspect of protein-protein interaction identified by biochemical or cell culture assays is the question of whether they have biological relevance. In particular, a common subcellular distribution pattern is an absolute necessity. In vitro studies presented here and in previous publications revealed promiscuous binding of Veli proteins to MAGUKs of the p55-subfamily (Butz et al., 1998; Kamberov et al., 2000; Tseng et al., 2001). The interactions are invariably mediated by the C-terminal of the two L27 modules of the p55-like MAGUKs which potentially heterodimerize with the single L27 domain located in the N-terminal region of the Veli proteins (Harris et al., 2002).

For the retina, we have shown that MPP4 and MPP5 colocalize with Veli3 isoforms at distinct intercellular junctions such as the photoreceptor synapse and the OLM region. By immunoprecipitation, we have isolated a complex consisting of MPP4 and Veli3 from solubilized retinal membranes providing confirmative evidence for a direct association between these two proteins. In contrast to the synaptic terminals in the OPL, Veli3 is expressed at a relatively low abundance in the OLM region, which likely accounts for the failure to immunoprecipitate significant amounts of native MPP5-Veli3 complexes from the retina. Alternatively, other proteins in the OLM region may compete with Veli3 for MPP5 binding. An interesting candidate would be the Veli1 isoform. The further analysis of putative Veli1 interactions with p55-like MAGUKs in the retina by immunohistochemistry or in vivo binding studies, however, is currently compromised by the lack of suitable Veli1 antibodies.

In brain synapses, a tripartite complex between Veli, CASK, and Mint1 is thought to play a role in synaptic vesicle exocytosis, synaptic adhesion, and protein targeting (Butz et al., 1998). Biochemical studies in epithelial cells have shown that protein-protein interaction between Veli and CASK is essential for the targeting of Veli to the basolateral surface (Straight et al., 2000), whereas binding to the PDZ domain of Veli seems to be responsible for the basolateral retention of transmembrane proteins, e.g., the epithelial GABA-transporter BGT-1 (Perego et al., 1999; Straight et al., 2001). In addition, Veli proteins appear to be part of multimolecular complexes that build the framework for adherens junctions in epithelia and neurons (Perego et al., 2000; Yamamoto et al., 2002). The colocalization of MPP4 and MPP5 with Veli3 at different sites of intercellular contacts in the mammalian retina suggests that the MAGUKs target Veli3 to different subcellular regions. Here, Veli3 may determine the accumulation of binding partners, thereby mediating the organization of retinal cell junctions. Interestingly, a recent study revealed that Drosophila lin-7 (Dlin-7) is recruited to specific membrane specializations by distinct MAGUK proteins (Bachmann et al., 2004). Std is responsible for the localization of Dlin-7 to the subapical region of epithelia and Discs-large (Dlg) for the postsynaptic localization of Dlin-7 at larval neuromuscular junctions.

In conclusion, our studies show that MPP4 and MPP5 are expressed at distinct subcellular sites of the mammalian retina and suggest specific functions of these closely related p55-like MAGUK proteins in this tissue. In addition, we have provided evidence for the existence of an association between Veli3 and the MAGUKs at different intercellular junctions of the retina, implying a role of the complexes in retinal cell adhesion and communication. The identification of additional proteins that bind Veli3, MPP4, and MPP5 in the retina will be crucial to further elucidate the role of these proteins in normal retinal function and to assess their contribution to retinal disease.

\section{LITERATURE CITED}

Bachmann A, Schneider M, Theilenberg E, Grawe F, Knust E. 2001. Drosophila Stardust is a partner of Crumbs in the control of epithelial cell polarity. Nature 414:638-643.

Bachmann A, Timmer M, Sierralta J, Pieterini G, Gundelfinger ED, Knust E, Thomas U. 2004. Cell type-specific recruitment of Drosophila Lin-7 to distinct MAGUK-based protein complexes defines novel roles for Sdt and Dlg-S97. J Cell Sci 117:1899-1909.

Bhat MA, Izaddoost S, Lu Y, Cho KO, Choi KW, Bellen HJ. 1999. Discs Lost, a novel multi-PDZ domain protein, establishes and maintains epithelial polarity. Cell 96:833-845.

Biedermann B, Bringmann A, Reichenbach A. 2002. High-affinity GABA uptake in retinal glial (Muller) cells of the guinea pig: electrophysiological characterization, immunohistochemical localization, and modeling of efficiency. Glia 39:217-228.

Butz S, Okamoto M, Sudhof TC. 1998. A tripartite protein complex with the potential to couple synaptic vesicle exocytosis to cell adhesion in brain. Cell 94:773-782.

den Hollander AI, ten Brink JB, de Kok YJ, van Soest S, van den Born LI, van Driel MA, van de Pol DJ, Payne AM, Bhattacharya SS, Kellner U, Hoyng CB, Westerveld A, Brunner HG, Bleeker-Wagemakers EM, Deutman AF, Heckenlively JR, Cremers FP, Bergen AA. 1999. Mutations in a human homologue of Drosophila crumbs cause retinitis pigmentosa (RP12). Nat Genet 23:217-221.

Dimitratos SD, Woods DF, Stathakis DG, Bryant PJ. 1999. Signaling pathways are focused at specialized regions of the plasma membrane by scaffolding proteins of the MAGUK family. Bioessays 21:912-921.

Fanning AS, Anderson JM. 1999. Protein modules as organizers of membrane structure. Curr Opin Cell Biol 11:432-439.

Gonzalez-Mariscal L, Betanzos A, Avila-Flores A. 2000. MAGUK proteins: structure and role in the tight junction. Semin Cell Dev Biol 11:315324.

Harris BZ, Venkatasubrahmanyam S, Lim WA. 2002. Coordinated folding and association of the LIN-2, -7 (L27) domain. An obligate heterodimerization involved in assembly of signaling and cell polarity complexes. J Biol Chem 277:34902-34908.

Hong Y, Stronach B, Perrimon N, Jan LY, Jan YN. 2001a. Drosophila Stardust interacts with Crumbs to control polarity of epithelia but not neuroblasts. Nature 414:634-638.

Hong DH, Yue G, Adamian M, Li T. 2001b. Retinitis pigmentosa GTPase regulator (RPGRr)-interacting protein is stably associated with the photoreceptor ciliary axoneme and anchors RPGR to the connecting cilium. J Biol Chem 276:12091-12099.

Hong DH, Pawlyk B, Sokolov M, Strissel KJ, Yang J, Tulloch B, Wright AF, Arshavsky VY, Li T. 2003. RPGR isoforms in photoreceptor connecting cilia and the transitional zone of motile cilia. Invest Ophthalmol Vis Sci 44:2413-2421.

Horst CJ, Johnson LV, Besharse JC. 1990. Transmembrane assemblage of the photoreceptor connecting cilium and motile cilium transition zone contain a common immunologic epitope. Cell Motil Cytoskeleton 17: $329-344$.

Izaddoost S, Nam SC, Bhat MA, Bellen HJ, Choi KW. 2002. Drosophila Crumbs is a positional cue in photoreceptor adherens junctions and rhabdomeres. Nature 416:178-183.

Jo K, Derin R, Li M, Bredt DS. 1999. Characterization of MALS/Velis-1, -2, and -3: a family of mammalian LIN-7 homologs enriched at brain 
synapses in association with the postsynaptic density-95/NMDA receptor postsynaptic complex. J Neurosci 19:4189-4199.

Kaech SM, Whitfield CW, Kim SK. 1998. The LIN-2/LIN-7/LIN-10 complex mediates basolateral membrane localization of the C. elegans EGF receptor LET-23 in vulval epithelial cells. Cell 94:761-771.

Kamberov E, Makarova O, Roh M, Liu A, Karnak D, Straight S, Margolis B. 2000. Molecular cloning and characterization of Pals, proteins associated with mLin-7. J Biol Chem 275:11425-11431.

Krämer F, Stöhr H, Weber BHF. 2004. Cloning and characterization of the murine Vmd2 RFP-TM gene family. Cytogenet Genome Res 105:107114.

Lemmers C, Medina E, Delgrossi MH, Michel D, Arsanto JP, Le Bivic A. 2002. hINADI/PATJ, a homolog of discs lost, interacts with crumbs and localizes to tight junctions in human epithelial cells. J Biol Chem 277:25408-25415.

Li M, Zhang SS, Barnstable CJ. 2003. Developmental and tissue expression patterns of mouse Mpp4 gene. Biochem Biophys Res Commun 307:229-235.

Linser PJ, Sorrentino M, Moscona AA. 1984. Cellular compartmentalization of carbonic anhydrase-C and glutamine synthetase in developing and mature mouse neural retina. Brain Res 315:65-71.

Lotery AJ, Jacobson SG, Fishman GA, Weleber RG, Fulton AB, Namperumalsamy P, Heon E, Levin AV, Grover S, Rosenow JR, Kopp KK, Sheffield VC, Stone EM. 2001. Mutations in the CRB1 gene cause Leber congenital amaurosis. Arch Ophthalmol 119:415-420.

MacKenzie D, Arendt A, Hargrave P, McDowell JH, Molday RS. 1984. Localization of binding sites for carboxyl terminal specific antirhodopsin monoclonal antibodies using synthetic peptides. Biochemistry 23:6544-6549.

Makarova O, Roh MH, Liu CJ, Laurinec S, Margolis B. 2003. Mammalian Crumbs 3 is a small transmembrane protein linked to protein associated with Lin-7 (Pals1). Gene 302:21-29.

Mavlyutov TA, Zhao H, Ferreira PA. 2002. Species-specific subcellular localization of RPGR and RPGRIP isoforms: implications for the phenotypic variability of congenital retinopathies among species. Hum Mol Genet 11:1899-1907.

Mehalow AK, Kameya S, Smith RS, Hawes NL, Denegre JM, Young JA, Bechtold L, Haider NB, Tepass U, Heckenlively JR, Chang B, Naggert JK, Nishina PM. 2003. CRB1 is essential for external limiting membrane integrity and photoreceptor morphogenesis in the mammalian retina. Hum Mol Genet 12:2179-2189.

Misawa H, Kawasaki Y, Mellor J, Sweeney N, Jo K, Nicoll RA, Bredt DS. 2001. Contrasting localizations of MALS/LIN-7 PDZ proteins in brain and molecular compensation in knockout mice. J Biol Chem 276:92649272.

Nam SC, Choi KW. 2003. Interaction of Par-6 and Crumbs complexes is essential for photoreceptor morphogenesis in Drosophila. Development 130:4363-4372.

Pfaffenholz R, Kuhn C, Grund C, Stehr S, Franke WW. 1999. The armrepeat protein NPRAP (neurojungin) is a constituent of the plaques of the outer limiting zone in the retina, defining a novel type of adhering junction. Exp Cell Res 250:452-464.

Pellikka M, Tanentzapf G, Pinto M, Smith C, McGlade CJ, Ready DF, Tepass U. 2002. Crumbs, the Drosophila homologue of human CRB1/
$\mathrm{RP} 12$, is essential for photoreceptor morphogenesis. Nature 416:143149.

Perego C, Vanoni C, Villa A, Longhi R, Kaech SM, Frohli E, Hajnal A, Kim SK, Pietrini G. 1999. PDZ-mediated interactions retain the epithelial GABA transporter on the basolateral surface of polarized epithelial cells. EMBO J 18:2384-2393.

Perego C, Vanoni C, Massari S, Longhi R, Pietrini G. 2000. Mammalian LIN-7 PDZ proteins associate with beta-catenin at the cell-cell junctions of epithelia and neurons. EMBO J 19:3978-3989.

Pielage J, Stork T, Bunse I, Klambt C. 2003. The Drosophila cell survival gene discs lost encodes a cytoplasmic Codanin-1-like protein, not a homolog of tight junction PDZ protein Patj. Dev Cell 5:841-851.

Rich KA, Zhan Y, Blanks JC. 1997. Migration and synaptogenesis of cone photoreceptors in the developing mouse retina. J Comp Neurol 388:4763.

Roh MH, Margolis B. 2003. Composition and function of PDZ protein complexes during cell polarization. Am J Physiol Renal Physiol 285: F377-387.

Roh MH, Makarova O, Liu CJ, Shin K, Lee S, Laurinec S, Goyal M, Wiggins R, Margolis B. 2002. The Maguk protein, Pals1, functions as an adapter, linking mammalian homologues of Crumbs and Discs Lost. J Cell Biol 157:161-172.

Stöhr H, Weber BH. 2001. Cloning and characterization of the human retina-specific gene MPP4, a novel member of the p55 subfamily of MAGUK proteins. Genomics 74:377-384.

Stöhr H, Stojic J, Weber BH. 2003. Cellular localization of the MPP4 protein in the mammalian retina. Invest Ophthalmol Vis Sci 44:50675074.

Straight SW, Karnak D, Borg JP, Kamberov E, Dare H, Margolis B, Wade JB. 2000 . mLin-7 is localized to the basolateral surface of renal epithelia via its $\mathrm{NH}(2)$ terminus. Am J Physiol Renal Physiol 278:F464-475.

Straight SW, Chen L, Karnak D, Margolis B. 2001. Interaction with mLin-7 alters the targeting of endocytosed transmembrane proteins in mammalian epithelial cells. Mol Biol Cell 12:1329-1340.

Tawara A, Varner HH, Hollyfield JG. 1988. Proteoglycans in the mouse interphotoreceptor matrix. I. Histochemical studies using cuprolinic blue. Exp Eye Res 46:689-704.

Tseng TC, Marfatia SM, Bryant PJ, Pack S, Zhuang Z, O’Brien JE, Lin L Hanada T, Chishti AH. 2001. VAM-1: a new member of the MAGUK family binds to human Veli-1 through a conserved domain. Biochim Biophys Acta 1518:249-259.

Tserentsoodol N, Shin BC, Suzuki T, Takata K. 1998. Colocalization of tight junction proteins, occludin and ZO-1, and glucose transporter GLUT1 in cells of the blood-ocular barrier in the mouse eye. Histochem Cell Biol 110:543-551.

Wei X, Malicki J. 2002. nagie oko, encoding a MAGUK-family protein, is essential for cellular patterning of the retina. Nat Genet 31:150-157.

Yamamoto Y, Mandai K, Okabe N, Hoshino T, Nakanishi H, Takai Y. 2002. Localization of mLin-7 at nectin-based cell-cell junctions. Oncogene 21:2545-2554.

Yan D, Swain PK, Breuer D, Tucker RM, Wu W, Fujita R, Rehemtulla A Burke D, Swaroop A. 1998. Biochemical characterization and subcellular localization of the mouse retinitis pigmentosa GTPase regulator (mRpgr). J Biol Chem 273:19656-19663. 